\title{
Quelle biodiversité pour quels habitants dans la trame verte urbaine ? L'exemple du Val Maubuée (Seine-et-Marne, France)
}

What Biodiversity for Which Inhabitants of the Urban Green Belt? The Example of Val Maubuée (Seine-et-Marne, France)

Laurent Simon et Lydie Goeldner-Gianella

\section{OpenEdition}

Journals

Édition électronique

URL : http://journals.openedition.org/developpementdurable/9326

DOI : 10.4000/developpementdurable.9326

ISSN : 1772-9971

Éditeur

Association DD\&T

Référence électronique

Laurent Simon et Lydie Goeldner-Gianella, «Quelle biodiversité pour quels habitants dans la trame verte urbaine? L'exemple du Val Maubuée (Seine-et-Marne, France) », Développement durable et territoires [En ligne], Vol. 3, n² 2 I Juillet 2012, mis en ligne le 06 juillet 2012, consulté le 19 avril 2019. URL : http://journals.openedition.org/developpementdurable/9326 ; DOI : 10.4000/ developpementdurable.9326

Ce document a été généré automatiquement le 19 avril 2019

Développement Durable et Territoires est mis à disposition selon les termes de la licence Creative Commons Attribution - Pas d'Utilisation Commerciale 4.0 International. 


\section{Quelle biodiversité pour quels habitants dans la trame verte urbaine ? L'exemple du Val Maubuée (Seine-et-Marne, France)}

What Biodiversity for Which Inhabitants of the Urban Green Belt? The Example

of Val Maubuée (Seine-et-Marne, France)

Laurent Simon et Lydie Goeldner-Gianella

\section{Introduction}

\subsection{Des enjeux de conservation face à l'érosion de la biodiversité des espaces périurbains}

Les espaces périurbains sont aujourd'hui au cœur de nombreux enjeux relatifs à la gestion-conservation de la biodiversité. Le premier enjeu majeur réside dans l'étalement urbain qui se fait souvent aux dépens d'espaces environnants plus riches en biodiversité (Wilcove et al., 1998; Wittemyer et al., 2008). L'urbanisation croissante est en effet considérée comme l'une des pressions majeures qui influence l'état de la biodiversité (Vitousek et al., 1997 ; Théobald, 2001 ; Teyssèdre, 2004 ) pouvant conduire à l'extinction de certaines espèces végétales et animales ou à la disparition de certains écosystèmes remarquables (Cincotta et al., 2000). Le département de Seine-et-Marne constitue de ce point de vue un terrain d'étude particulièrement fécond. Territoire de biodiversité reconnu avec des espaces labellisés, certains de niveau international comme la forêt de Fontainebleau, ainsi que de nombreux espaces de biodiversité ordinaire (forêts, zones humides...), il est depuis 50 ans confronté à des dynamiques d'urbanisation fortes (Berger, 2004), qui se traduisent notamment par une diminution des habitats «naturels » et par une fragmentation accrue des paysages (Mimet et al., 2011). La biodiversité y est donc fortement menacée : diminution des espèces rares et autochtones au profit d'espèces 
généralistes et invasives, réduction des habitats et fragmentation (Devictor et alii, 2008; Filoche et al., 2010) et peut être considérée localement comme résiduelle. Le maintien d'espaces de connectivité écologique y est ainsi considéré comme un défi majeur dont témoignent les documents d'aménagement (SDRIF, $\mathrm{SCOT}^{1}$ ) récents. Notre travail portera à la fois sur des espaces identifiés comme des zones potentielles de connectivité biologique, justifiant ainsi le terme de corridor (c'est le cas du corridor des étangs) et sur des espaces assimilés à de simples éléments de la trame verte entendue comme une continuité paysagère et végétale (c'est le cas de l'Allée des bois que nous dénommerons «liaison verte RER»). Si les espaces de connectivité sont censés permettre le maintien de la biodiversité entendue non seulement sous l'angle de sa richesse spécifique et écosystémique mais aussi sous l'angle de son fonctionnement (possibilité de déplacement des espèces, interaction entre écosystèmes...), les espaces de trame verte apparaissent en revanche avant tout comme des infrastructures urbaines destinées au bien-être des habitants.

\subsection{Un enjeu social autant qu'écologique}

2 Si l'enjeu écologique parait indéniable, les questions sociales se posent aussi : celle de l'usage et donc de l'accessibilité de ces espaces de connexion, celle des bénéfices ressentis par les habitants, celle enfin de l'acceptabilité sociale des politiques de trame verte et bleue engagées. La prise en compte de ces deux types d'espaces (corridor biologique et éléments de trame verte) permet de s'interroger sur le rôle de la biodiversité dans la perception qu'ont les citoyens de ces espaces. Dans un article de synthèse Dearborn et Kark (2010) ont souligné les raisons qui conduisent aujourd'hui à vouloir préserver des espaces de biodiversité en milieu urbain. Aux motivations écologiques (services de régulation et de support) s'ajoutent des motivations sociales liées notamment au bienêtre (well-being en anglais ${ }^{2}$ ) que procureraient ces espaces de biodiversité. Toutefois peu de travaux ont réellement démontré le lien entre biodiversité (et pas seulement présence d'espaces verts) et sentiment de bien-être. Les travaux de Fuller et al. (2007) sont parmi les seuls qui s'interrogent sur le lien entre accroissement de la diversité spécifique et sentiment de bien-être dans des espaces verts urbains. Le travail que nous présentons ne prétend pas apporter de réponse formelle à cette question du lien entre biodiversité et bien-être. Il cherche en revanche à voir dans quelle mesure la biodiversité réellement présente dans les corridors participe de l'attachement des citadins à ces espaces. Le choix de trois espaces différenciés du point de vue de leur qualité écologique (corridor biologique d'un côté, continuité verte de l'autre) répond à cette interrogation.

\subsection{Choix du terrain et problématique}

Une première enquête réalisée dans quatre communes urbaines et périurbaines du département (Simon et al., 2012) avait notamment souligné le rôle des espaces et des acteurs de proximité dans les processus de prise de conscience par les habitants de l'importance de la biodiversité, par ailleurs rarement perçue comme un enjeu majeur. Cette même enquête mettait en évidence les inégalités sociales qui existent dans la connaissance de la biodiversité et surtout dans l'accès aux espaces de biodiversité « ordinaires » (bois, étangs...) de proximité. Aux catégories socioprofessionnelles les plus favorisées et les mieux connectées à la biodiversité de proximité s'opposait un ensemble 
de population plutôt jeune, peu diplômée, vivant le plus souvent en habitat collectif avec des revenus significativement plus bas que la moyenne.

Dès lors se pose la question de la mise en place de ces trames vertes urbaines. Le choix que nous avons fait du Val Maubuée (fig. 1) s'explique en premier lieu par son appartenance à la ville nouvelle de Marne-la-Vallée, créée dans les années soixante à l'Est de Paris avec la volonté de combiner urbanisation et conservation d'espaces naturels à des fins paysagères et sociales, pour en faire une "cité jardin linéaire» (Eleb-Harlé et Barles, 2005). C'est ainsi que 1700 ha d'espaces verts - soit $200 \mathrm{~m}^{2}$ d'espaces verts par habitant - sont restés préservés dans le Val Maubuée, situé à la jonction de l'autoroute A4 et de la francilienne. Comme le dit un élu, interrogé par M.-J Menozzi (2011) : «au Val Maubuée, c'est la ville qui est venue s'installer dans la nature, pas le contraire».

Figure 1. Localisation de la zone d'étude en région parisienne (Les corridors étudiés sont situés au Val Maubuée. Cf. fig.2)

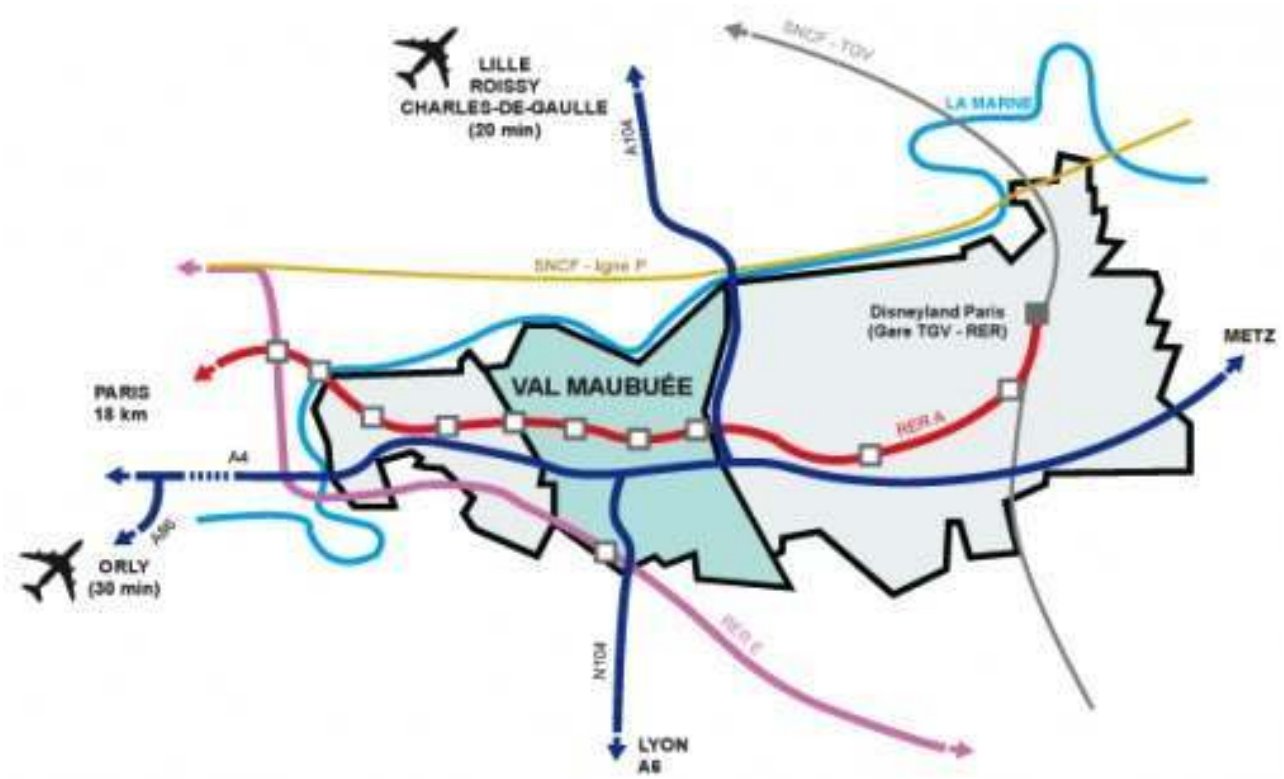

Source : site internet du SAN Val Maubuée (http://www.valmaubuee.fr/agglomeration/situation/)

Ce choix du Val Maubuée, composée de six communes (cf. fig. 2), s'explique aussi par l'implication forte des élus de ce Syndicat d'Agglomération Nouvelle (SAN) dans les politiques environnementales en général, dans celles liées à la biodiversité en particulier. La participation du SAN au programme European Landscapes Management (Euroscapes ${ }^{3}$ ), la mise en place actuelle d'un SCOP (Schéma de Cohérence et d'Orientation Paysagère), la place enfin accordée aux continuités vertes et écologiques dans le SCOT à la suite notamment d'un diagnostic écologique attestent de cette implication. Un scénario de développement du Val Maubuée, arrêté par le SAN pour les 20 ans à venir, doit justement favoriser la préservation et la connexion des espaces verts et naturels structurants, qui représentent $40 \%$ du territoire.

Dans un tel contexte, il nous a semblé important, dans le cadre du programme «ANR trame verte urbaine » (Blanc N., Clergeau P, 2010)), de prolonger l'enquête menée précédemment en nous interrogeant sur les éventuelles spécificités des espaces 
constitutifs de la trame verte aux yeux des habitants. Plusieurs questions sont ainsi posées dans cet article à partir du cas du Val Maubuée :

- Les « corridors ${ }^{4}$ » sont-ils des espaces valorisés par les habitants et vis-à-vis desquels ils témoigneraient, par leurs pratiques et leurs usages d'un véritable attachement?

- Quelle est la part de la biodiversité dans cet attachement ? L'espace est-il valorisé en tant qu'espace public, en tant qu'espace vert ou en tant qu'espace doté d'une véritable biodiversité?

- Les différences sociales dans l'usage et la perception des corridors sont-elles importantes et reproduisent-elles les inégalités recensées dans la première enquête?

7 Ces questions permettent d'introduire une discussion plus générale sur l'acceptabilité de ces politiques de trame verte et la possibilité d'en faire des outils de connexion à la biodiversité pour des catégories sociales qui en général y ont peu accès (Simon L.et al, 2012, Shwartz A. et alii, 2012).

\section{Lieux d'étude et méthodologie}

\subsection{Le choix de trois corridors d'un intérêt écologique inégal}

Trois espaces ont ainsi été identifiés pour répondre à ces questions (cf. fig. 2). Les principaux paramètres qu'il convenait de moduler tenaient à la qualité écologique des corridors et aux enjeux identifiés et labellisés en matière de biodiversité remarquable (Zone Naturelle d'Intérêt Ecologique Faunistique et Floristique - ZNIEFF -, espèces rares...) d'une part, aux caractéristiques sociales des usagers d'autre part.

Concernant la biodiversité effectivement présente, le diagnostic écologique réalisé par le bureau d'étude ECOSPHERE distingue clairement les trois corridors choisis (fig. 2) : le corridor des étangs fait figure d'espace possédant une valeur écologique (floristique, faunistique et habitats) moyenne à assez forte (richesse et abondance batrachologique, diversité floristique et habitats remarquables), alors que le corridor de champs n'est identifié que comme un corridor potentiel à faible valeur écologique et le « corridor » de l'allée des bois («liaison verte RER») n'est même pas considéré comme pouvant s'intégrer aux continuités écologiques du SAN. Au plan social, ces communes présentent des contrastes assez importants le long des corridors, les prix de l'immobilier pouvant varier d'une fourchette de $2430-2560 € / \mathrm{m}^{2}$ à une fourchette de $3070-3210 € / \mathrm{m}^{2}$, selon les estimations du site «MeilleursAgents.com » sur la base des ventes enregistrées par les Notaires de Paris / Ile-de-France (base BIEN) et le réseau des agences immobilières partenaires ${ }^{5}$. On constate toutefois que la proximité des corridors ne se traduit pas nécessairement par les prix les plus élevés, notamment dans les communes de Champssur-Marne ou Torcy, où ceux-ci peuvent dépasser $3610 € / \mathrm{m}^{2}$ dans d'autres quartiers. 
Figure 2 : les corridors étudiés au sein de la trame verte du Val Maubuée

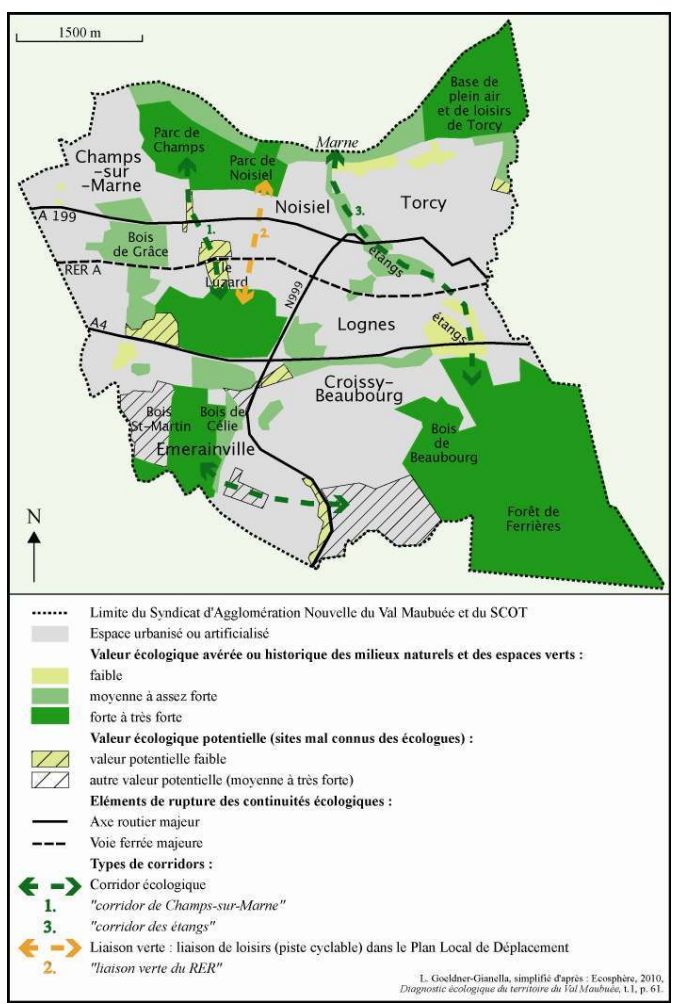

10 Le corridor de Champs, situé en face du Château, appartient au domaine du château. Il s'agit d'un espace de pelouse bordé d'arbres caducifoliés (photo 1). Des massifs de plantes rudérales «spontanées»y coexistent avec une pelouse entretenue. Le diagnostic écologique réalisé sur le site par le bureau d'étude «Ecosphère » n'y mentionne que peu de potentialités floristiques mais souligne la présence de chiroptères relativement rares dont trois pipistrelles. L'étude souligne l'intérêt potentiel du site comme corridor écologique reliant le parc de champ au bois de Grange - deux espaces considérés comme à forte ou très forte biodiversité. Il s'agit de l'un des deux corridors clairement identifiés dans le SCOT du Val Maubuée (cf. fig. 2). Ce corridor est interrompu par l'ancienne autoroute A 199 que franchit ici un pont relativement étroit. Le quartier environnant du vieux Champs est habité par une population relativement aisée avec une assez forte proportion de cadres supérieurs ( $21 \%$ de la population active, pour $28 \%$ d'employés et $16 \%$ d'ouvriers). L'habitat individuel y domine avec 57,8 \% des logements. Dans la partie nord du corridor, à proximité du château, les prix immobiliers sont les plus élevés des secteurs longeant les trois corridors, se situant dans une fourchette allant de 3210 à 3610 $€ / \mathrm{m}^{2}$. Par contre ils chutent fortement au sud de l'A199, n'étant plus compris, en moyenne, qu'entre 2680 et $2800 € / \mathrm{m}^{2}$. 
Photographie 1 (L. Goeldner-Gianella) : le corridor de Champs-sur-Marne

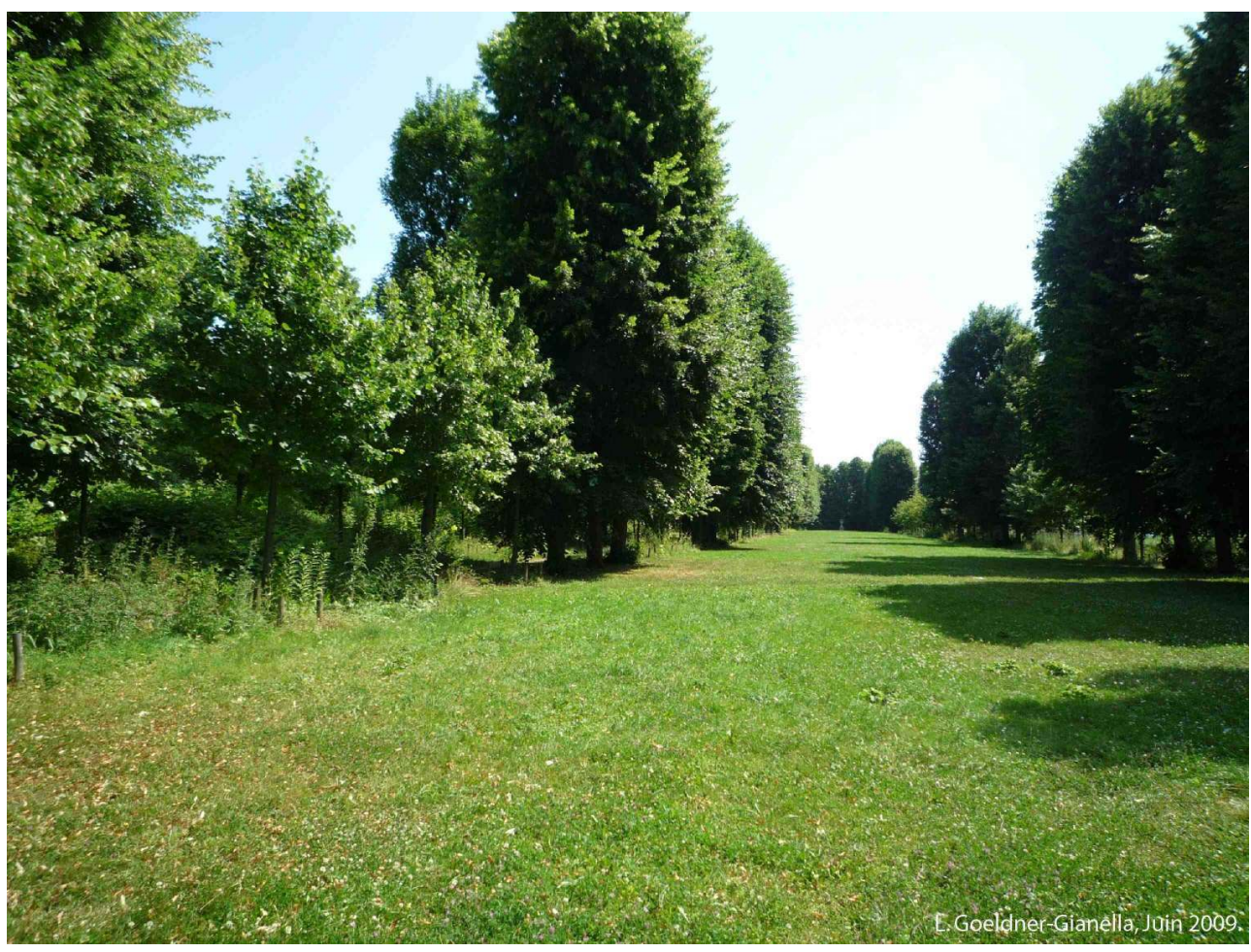

11 L'allée des bois, appelée « liaison verte RER » relie le parc de Noisiel au bois de la Grange au sud (deux espaces situés en ZNIEFF1). Il s'agit d'une allée piétonne doublée d'une piste cyclable, caractérisée par des alignements d'arbres à dominante de feuillus, des petits massifs d'arbustes et des pelouses aménagées. L'espace joue certes le rôle de liaison verte entre des espaces de fort potentiel biologique mais son aménagement actuel est encore limité du point de vue écologique, et les coupures fortes dues aux infrastructures routières en font davantage un espace de liaison douce centré sur le RER qu'un réel corridor écologique. L'Allée des bois constitue ainsi davantage un élément de la trame verte (une liaison verte de loisirs identifiée dans le Plan Local de Déplacements du Val Maubuée) qu'un espace de connexion biologique (cf. fig. 2). Cet espace s'insère dans un tissu urbain où dominent les habitats collectifs plus ou moins élevés (photo 2), habités par une population aux revenus plus modestes $(11,6 \%$ de cadres supérieurs au sein de la population active pour $33 \%$ d'employés et $25 \%$ d'ouvriers). Les prix immobiliers, compris en moyenne entre 2430 et $2560 € / \mathrm{m}^{2}$, soulignent cet écart avec le corridor de Champs. 
Photographie 2 (L. Goeldner-Gianella) : le corridor du RER

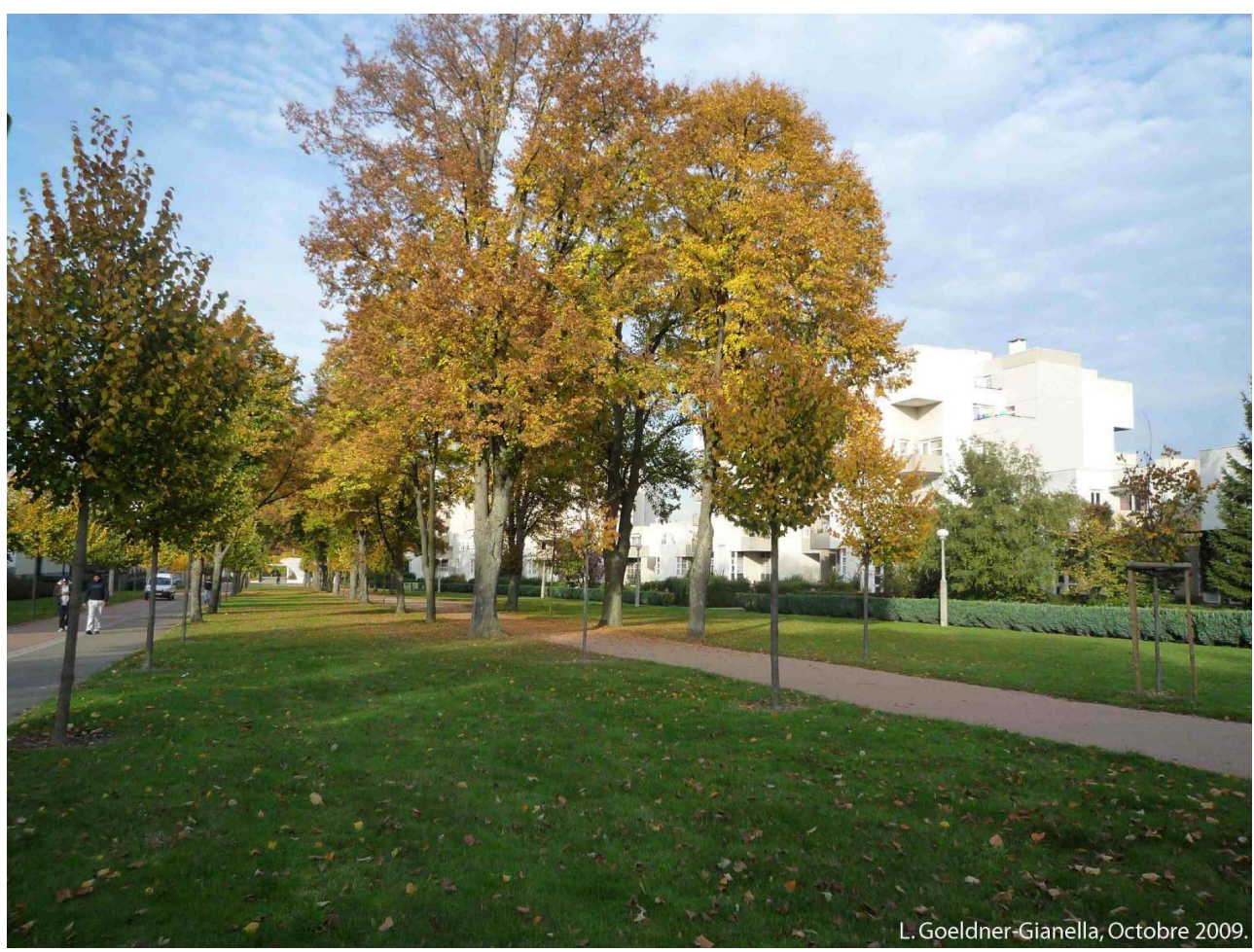

Le dernier corridor, dénommé " corridor des étangs », relie l'extrémité nord de la forêt de Ferrières, principal noyau de biodiversité autour de la ville nouvelle, aux espaces boisés de Croissy-Beaubourg et des rives de la Marne entre Noisiel et Torcy. L'espace est ici partagé entre de vastes étangs parfois bordés de roselières, des pelouses et des paysages arborés (photo 3). C'est le deuxième corridor écologique clairement identifié dans le SCOT du Val Maubuée. L'étude du Bureau Ecosphère le considère comme ayant une valeur écologique moyenne à assez forte (fig. 2), avec des enjeux de biodiversité importants aussi bien en termes d'habitats que d'espèces faunistiques ou floristiques. S'étendant sur près de 4 kilomètres du Nord au Sud, coupé par de nombreuses infrastructures de transport (voies urbaines majeures, autoroutes et RER), il traverse des quartiers aux caractéristiques sociales contrastées. En effet, le long du corridor, les prix de l'immobilier présentent des fourchettes très variées, d'un minimum, à Lognes, de $2430 € / \mathrm{m}^{2}$ à un maximum, au nord des communes de Noisiel et Torcy, près de la Marne, de $3210 € / \mathrm{m}^{2}$. 
Photographie 3 (L. Goeldner-Gianella) : le corridor des étangs

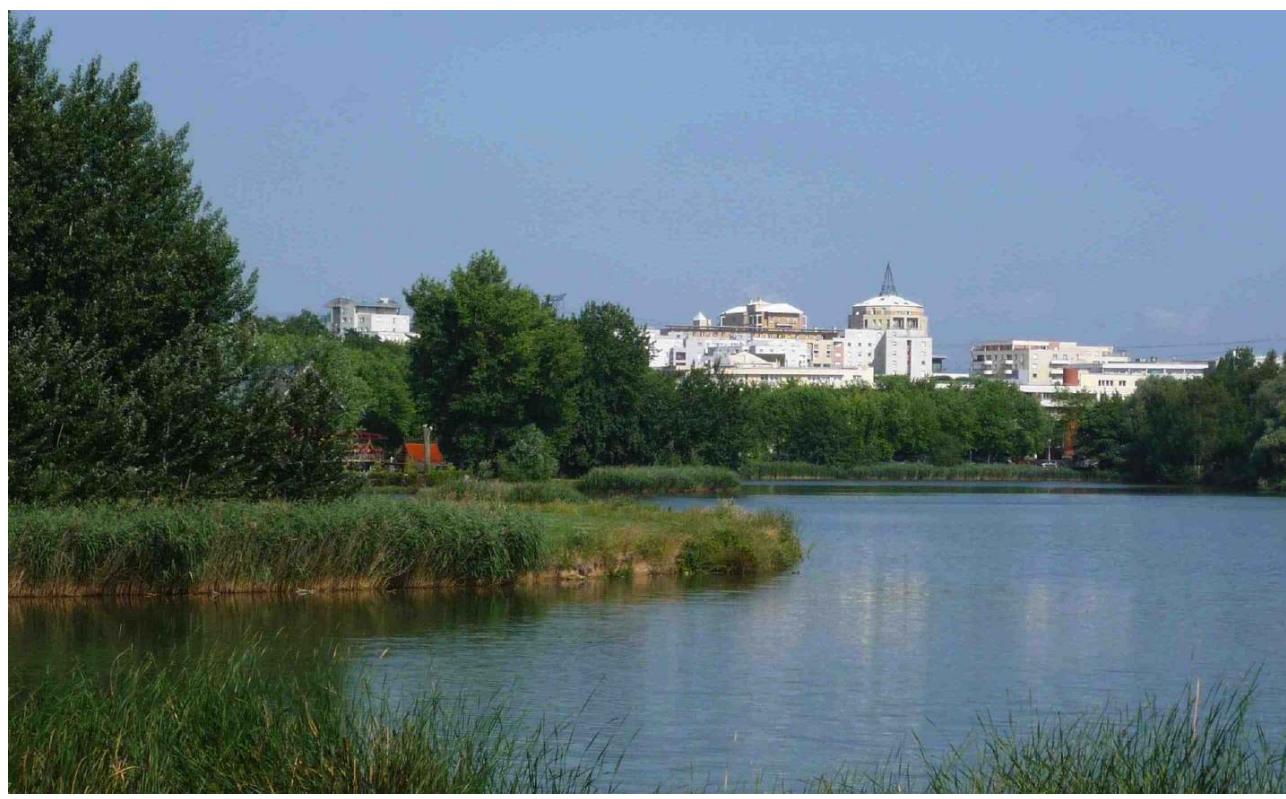

Il convient donc de relativiser l'usage ici du terme de corridor. Si deux au moins de ces espaces sont bien identifiés comme des zones de connexion biologique existantes et qu'il convient de renforcer, tous ces espaces sont également fragmentés et d'un intérêt biologique inégal.

\subsection{Méthodes d'étude}

Pour répondre à notre questionnement etpour aider à la constitution d'un questionnaire destiné aux usagers, 24 entretiens préliminaires ont été réalisés dans les corridors à l'automne 2008, afin de tester le vocabulaire à utiliser dans le questionnaire, et préparer son contenu et la formulation des questions. Ces entretiens, souvent longs (d'une durée de $30 \mathrm{mn}$ à $1 \mathrm{~h} 30$ ), sont en soi une riche source d'information sur le sujet, pouvant aider à une meilleure compréhension des résultats quantitatifs (cf. 3.1.2.).

Le questionnaire a porté dans une première partie sur des questions de fréquentation, d'usages, de perceptions sensorielles et de représentations des corridors. Il a consisté, dans une seconde partie, à évaluer le consentement à payer des usagers, en les interrogeant sur le montant d'un don, d'un ticket d'entrée ou de stationnement ou d'un supplément d'impôts locaux qu'ils seraient prêts à payer pour l'entretien et la protection des corridors. Enfin, la partie concernant le profil personnel des individus a également permis - outre des questions usuelles - d'en savoir plus sur leur parcours résidentiel entre ville et campagne, la distance à pied entre leur lieu de résidence et le corridor, et leur sensibilité à la nature ou à l'environnement (à travers des questions sur leurs loisirs, leurs lectures et leur appartenance associative). La passation des questionnaires a été réalisée entre l'automne 2009 et le printemps 2010. Les 534 personnes interrogées se répartissent de façon relativement équilibrée entre les trois corridors : $29.7 \%$ des usagers ont été interrogés dans le corridor de Champs-sur-Marne, $41.6 \%$ dans celui du RER et $28.7 \%$ dans celui des étangs. 
Pour intégrer ces enquêtes à un système d'information multi-sources plus complet, permettant de mieux les interpréter, d'autres entretiens sont prévus avec les acteurs de l'environnement et de l'aménagement du secteur d'étude. Par ailleurs, le recours à des statistiques démographiques et géographiques ou à des données écologiques a permis des comparaisons entre la réalité écologique du terrain et les perceptions des usagers, de même qu'entre les usagers des corridors et les riverains proches (dans le cadre des IRIS ${ }^{6}$ ), afin d'affiner notre compréhension sociale de ces espaces. Des comptages d'usagers ont également été réalisés au printemps 2010. Ceux-ci restent toutefois partiels car il est impossible de réaliser des comptages exhaustifs dans ces corridors, tant les possibilités d'entrée sont nombreuses depuis les résidences individuelles et collectives qui les jouxtent ou les rues et sentiers qui y mènent ${ }^{7}$.

17 Le tableau suivant (tab. 1) synthétise les principaux résultats de l'enquête, pour la population totale et par corridor - dès lors que les croisements de variables se sont avérés significatifs au seuil de $5 \%$ (test du khi 2).

Tableau 1 : caractéristiques, activités, consentement à payer et perceptions des usagers des trois corridors

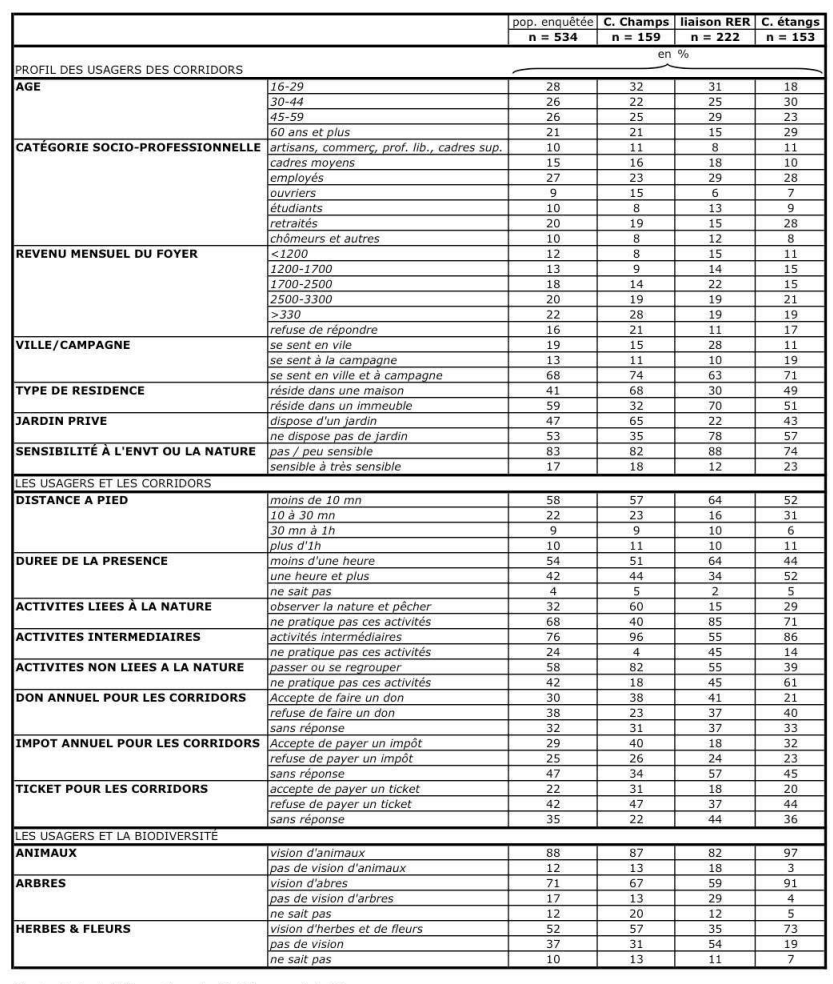

$\mathrm{Nb}$ : les tests du khiz sont tous significatiff au seul de $5 \%$.

\section{Résultats}

\subsection{Les corridors : des espaces appréciés de tous}

\subsubsection{Des espaces très fréquentés}

Les enquêtes menées auprès des usagers font ressortir plusieurs caractéristiques importantes touchant à la fréquentation des corridors. La première tient au caractère de proximité de cette fréquentation. $80 \%$ des enquêtés habitent à moins de 30 minutes à 
pied du corridor, et même 50 à $60 \%$ de l'échantillon à moins de dix minutes, quel que soit le corridor considéré. Ce sont donc bien très majoritairement les habitants des communes concernées qui fréquentent ces corridors, comme une analyse géographique l'a montré par ailleurs (Lardinois, 2011), et qui y viennent à pied ${ }^{8}$.

L'espace est ainsi investi par des usagers qui reflètent assez largement la composition sociale des quartiers (saisis au niveau des IRIS) environnants. Dans l'ensemble des corridors, ce sont en définitive les catégories moyennes (employés notamment) qui apparaissent surreprésentées par rapport à la population des communes environnantes (tableau 2). Les catégories sociales les plus aisées et les moins aisées ne fréquentent pas particulièrement les corridors (notre échantillon d'usagers compte respectivement $10 \%$ d'artisans/commerçants, professions libérales et cadres et $9 \%$ d'ouvriers).

Tableau 2 : répartition des CSP dans les IRIS voisins des corridors de Champs-sur-Marne et du RER et dans l'ensemble du Val Maubuée

\begin{tabular}{|c|c|c|c|c|c|c|}
\hline & CSP & \multirow{2}{*}{$\begin{array}{c}\text { Artisans et } \\
\text { commerçants }\end{array}$} & \multirow{2}{*}{$\begin{array}{c}\text { Cadres } \\
\text { supérieurs }\end{array}$} & \multirow{2}{*}{$\begin{array}{c}\text { Professions } \\
\text { intermédiaires }\end{array}$} & \multirow{2}{*}{ Employés } & \multirow{2}{*}{ Ouvriers } \\
\hline & IRIS & & & & & \\
\hline $\begin{array}{l}\text { Proximité du } \\
\text { corridor de } \\
\text { Champs-sur- }\end{array}$ & IRIS 2 & 5.1 & 21 & 29.9 & 28 & 16 \\
\hline & IRIS 21 & 0.7 & 11.6 & 29.8 & 32.9 & 25 \\
\hline & IRIS 4 & 3.1 & 33 & 26.8 & 28.5 & 8.6 \\
\hline $\begin{array}{l}\text { Proximité de } \\
\text { la liaison verte } \\
\text { RER }\end{array}$ & IRIS 22 & 5.4 & 13 & 21.4 & 37.2 & 23 \\
\hline & IRIS 21 & 0.7 & 11.6 & 29.8 & 32.9 & 25 \\
\hline & IRIS 19 & 1.9 & 25.4 & 24.3 & 35.7 & 12.8 \\
\hline $\begin{array}{l}\text { Ensemble du } \\
\text { Val Maubuée }\end{array}$ & & 3.6 & 17.6 & 28.6 & 33.4 & 16.7 \\
\hline
\end{tabular}

20 Si l'on cherche à appréhender les caractéristiques sociales des personnes fréquentant le corridor par le niveau de diplôme, on constate peu de différence avec la population environnante, rien en tout état de cause qui puisse être vraiment significatif (légère surreprésentation des hauts diplômes dans deux corridors sur trois, sous-représentation dans le troisième). Dernier aspect intéressant : si les populations âgées de 60 à 74 ans sont certes surreprésentées dans les corridors par rapport à la population des communes voisines ${ }^{9}$, le déséquilibre entre classes d'âge reste limité, les populations jeunes (16-29 ans) constituant $28 \%$ des usagers des trois corridors alors qu'ils représentent autour de $30 \%$ dans les communes de Torcy, Noisiel et Champs.

Tableau 3 : répartition par âge (pour la population 15 ans et +) dans les communes et dans les corridors

\begin{tabular}{|l|c|c|c|c|c|}
\hline Classes d'âge & $\mathbf{1 5 - 2 9}$ & $\mathbf{3 0 - 4 4}$ & $\mathbf{4 5 - 5 9}$ & $\mathbf{6 0 - 7 4}$ & $\mathbf{7 5}$ et + \\
\hline Communes & 29.5 & 27.5 & 28.8 & 10.4 & 3.8 \\
\hline Noisiel & 29.8 & 28.1 & 28 & 10.4 & 3.6 \\
\hline Champs & 31.4 & 28.5 & 26.2 & 9.6 & 4.2 \\
\hline Torcy & 27.8 & 25.5 & 25.9 & 17.6 & 3.1 \\
\hline 3 corridors &
\end{tabular}

21 Au final, les corridors par leur dimension d'espaces de proximité n'apparaissent pas (ou très peu) comme des espaces de ségrégation sociale et générationnelle ce qui les différencie nettement des espaces de biodiversité « hors la ville » étudiés lors de l'enquête menée précédemment dans quatre communes de Seine-et-Marne. C'est une donnée à 
notre avis fondamentale pour toute politique qui voudrait associer accès à la biodiversité et justice sociale.

Cette fréquentation ${ }^{10}$ répond-elle pour autant à un réel besoin ou n'est-elle qu'une utilisation de circonstance ? Le résultat des enquêtes montre que pour près d'un enquêté sur deux ( $42 \%)$ la durée de fréquentation est supérieure à 1 heure et ne correspond donc pas un à simple passage. Même dans le corridor RER, plus d'un tiers des enquêtés passe 1 heure ou plus. Davantage encore que la durée, c'est le rythme de fréquentation qui atteste d'un réel intérêt pour ces espaces. Quel que soit le corridor, les enquêtés affirment à plus de $80 \%$ s'y rendre toutes les semaines.

Ce premier ensemble de remarques souligne ainsi deux aspects qui nous semblent importants dès lors que l'on souhaiterait envisager des politiques d'accès à la biodiversité en ville. Les corridors choisis permettent en effet de toucher une large population, sans réelle distinction d'âge ou de niveau social, et dont l'usage du corridor dépasse de loin le simple passage.

\subsubsection{Un attachement qui dépasse les clivages sociaux}

24 Cette fréquentation signifie-t-elle pour autant que les enquêtés apprécient le corridor? Les entretiens préliminaires laissaient déjà percevoir l'attachement des personnes interrogées: "c'est un mini paradis, surtout en ce moment avec toutes ces couleurs"; "C'est l'idéal pour une personne à la retraite, c'est tranquille»; le bien-être, la paix, la tranquillité»; « il faut que ça reste tel que c'est là»; "magnifique, calme»; "je me sens dans un environnement sain, je respire mieux, je me sens plus à l'aise.» - autant d'expressions qui reviennent fréquemment et traduisent un sentiment de "bien-être ». Les enquêtes quantitatives viennent confirmer l'impression laissée par les entretiens préliminaires. A la question « si vous deviez définir cet endroit par trois mots que diriez-vous?» les enquêtés répondent très majoritairement par des qualificatifs positifs associés au calme (35\%), au vert (12\%), à la beauté $(10 \%)$ et à la convivialité $(6 \%)$. Les notions à connotations négatives (" triste » "moche", "sale» "déprimant» "bruyant») ne totalisent que moins de $5 \%$ des réponses. Près de $82 \%$ des enquêtés se disent d'ailleurs concernés par la protection de ces espaces.

Pour mieux évaluer cet attachement aux corridors sélectionnés plusieurs questions portaient sur une éventuelle participation financière à leur entretien et à leur protection, soit sous forme d'un don, soit sous forme d'un ticket de passage soit enfin sous la forme d'une augmentation d'impôts locaux. Les résultats obtenus mettent en évidence trois points essentiels : la proportion d'enquêtés se déclarant prêts à verser quelque chose sous une forme ou sous une autre est loin d'être négligeable : $30 \%$, acceptent de faire un don annuel ${ }^{11}, 22 \%$ de payer un ticket ${ }^{12}$ et $29 \%$ une augmentation d'impôt étalée sur cinq ans ${ }^{13}$. Le refus de participer n'est que rarement lié à un désintérêt pour le lieu (« il n'y a pas besoin de protéger et d'entretenir cet endroit ») mais plutôt à l'idée que ce n'est pas aux usagers de payer et/ou que les impôts sont déjà destinés à l'entretien de ces espaces. Enfin la composition sociale des donneurs potentiels est intéressante: si les "hauts » revenus sont plus réceptifs à l'idée d'une participation financière sous forme de don (38\% des revenus supérieurs à 3300 euros/mois contre $27 \%$ des revenus inférieurs à 1200 euros/mois) ou d'augmentation d'impôt (respectivement $40 \%$ contre $14 \%$ ), les populations à bas revenus consentent davantage à payer un ticket $(33 \%$ contre $24 \%$ cette fois) et surtout le montant de leur participation, si on le rapporte à leurs revenus, est 
dans tous les cas (dons, tickets ou impôts) supérieur à celui proposé par les populations plus aisées. Pour ne prendre que le cas du don, les revenus supérieurs à 2700 euros/mois envisagent (pour ceux qui acceptent de payer) un don annuel moyen de 26,3 euros alors que les revenus inférieurs à 1200 euros/mois envisagent un don moyen de 17 euros.

L'enquête réalisée confirme ainsi deux points essentiels dans la compréhension du rôle social des corridors étudiés : un fort attachement d'ensemble des usagers lié aux services que procurent ces espaces et une attractivité proportionnellement plus forte auprès de populations peu aisées - dont l'enquête précédente avait pourtant souligné la faible connexion à la biodiversité (Simon et al, 2012).

\section{2. Les corridors : des espaces de connexion à la biodiversité ?}

Si l'attachement à la trame verte se dessine ainsi clairement, doit-on pour autant les considérer comme des espaces où la biodiversité joue un rôle et pourrait être ainsi valorisée, auprès notamment de populations qui autrement n'y ont pas ou peu accès ?

\subsubsection{Des espaces qui rendent essentiellement des « services culturels »...}

L'idée centrale qui se dégage des enquêtes, et qui n'est en rien surprenante, est celle d'une très faible prise en compte de la biodiversité par les usagers des "corridors ». Parmi les mots choisis par les usagers pour décrire ces espaces le mot nature n'est que rarement cité $(3,5 \%)$. Si le mot « vert » revient un peu plus souvent $(11,3 \%)$ il reflète autant sinon davantage un sentiment paysager d'ensemble qu'une attention portée à la diversité du vivant. Considérant les éléments les plus importants dans ces espaces, les caractéristiques liées à la biodiversité, au sens écologique, sont largement minoritaires («arbres» et « eau » ne sont cités que par moins de $20 \%$ de enquêtés) par rapport aux notions relatives aux services culturels ("tranquillité », «oisirs»...) et guère plus importantes que celles de simple confort (" proximité » et "propreté »). Les suggestions proposées pour améliorer ces espaces ne font d'ailleurs jamais appel à la biodiversité voire contribuent potentiellement à la limiter («tondre les pelouses»). Les souhaits majoritaires tournent autour de la propreté de l'espace vert, de l'amélioration des services («plus de bancs, plus de poubelles») et de l'offre d'activités de loisirs. Les activités pratiquées dans ces espaces sont d'ailleurs très largement étrangères à la biodiversité. A la question "quelles activités avez-vous fait ou allez-vous faire sur le site?" " plusieurs réponses étant possibles, seuls 169 enquêtés (32\%) sur 530 répondent "pour observer la nature ». Ils sont près de $60 \%$ à y venir pour se balader, $50 \%$ pour simplement "y passer », $30 \%$ pour s'asseoir ou pour jouer. Il est intéressant de noter que ceux qui viennent explicitement pour «observer la nature» ne se distinguent pratiquement pas en proportion de ceux qui ne viennent pas à cette fin: ni par leur appartenance sociale (CSP), ni pas leur degré de fréquentation, ni par leur perception de la faune. Ils sont néanmoins plus nombreux à voir des plantes et à être favorables à la protection des corridors et sont globalement plus âgés. On ne retrouve toutefois pas dans les corridors une coupure sociale aussi franche que celle que nous avions observée dans l'enquête sur les citadins en Seine-et-Marne et qui opposait les " habitants ordinaires » à des habitants tout particulièrement intéressés par la biodiversité. Au vu de ces premiers résultats la biodiversité, dans sa dimension proprement écologique, n'est guère perçue de manière consciente comme un élément important et attractif des espaces considérés. 


\subsection{2. ... et où le sentiment de continuité est faible}

Dans la mesure où ces espaces pourraient être destinés à favoriser les continuités écologiques, il nous a semblé important de nous interroger sur la perception que peuvent avoir les habitants quant à l'existence ou non d'une trame verte ou plus simplement d'une cohérence de ces espaces de connectivité. Les notions même de trame verte et plus encore de corridor écologique sont largement méconnues des enquêtés. Moins de $5 \%$ d'entre eux déclarent connaître les deux expressions et la notion de trame verte, la moins étrangère, n'est connue que par 10 \% d'entre eux et correctement définie par moins de $5 \%$. continuité que leurs usagers ressentent ou non. L'analyse des réponses à la question «pour vous, où commence et où finit » ce corridor montre que cette continuité n'est en majorité perçue que partiellement, pour ceux de Champs et du RER, voire non perçue ou difficile à définir pour celui des étangs - au sujet duquel $75 \%$ des réponses sont imprécises ou hors sujet. Il n'existe donc pas de connaissance ou de représentation d'une continuité totale ou quasi-totale entre les tâches d'habitat que relient ces corridors ${ }^{14}$ (cf. fig. 2). Des continuités partielles sont toutefois fréquemment citées, notamment pour les parties nord du corridor du RER (au nord de la station) et sud du corridor de Champs (au sud de l'A199).

Tableau 4 : Degré de perception de la continuité des corridors (en \% du nombre de répondants par corridor).

\begin{tabular}{|c|c|c|c|}
\hline & $\begin{array}{c}\text { Limites imprécises } \\
\text { ou hors-sujet }\end{array}$ & $\begin{array}{c}\text { Limites nord/sud } \\
\text { trop rapprochées }\end{array}$ & $\begin{array}{c}\text { Perception d'une } \\
\text { continuité partielle } \\
\text { au sud }\end{array}$ \\
\hline $\begin{array}{c}\text { Corridor de } \\
\text { Champs }\end{array}$ & 6 & $/$ & 28 \\
\hline $\begin{array}{c}\text { Corridor du } \\
\text { RER }\end{array}$ & 10,5 & 3 & 9 \\
\hline $\begin{array}{c}\text { Corridor des } \\
\text { étangs }\end{array}$ & 75 & 13 & $<2$ \\
\hline
\end{tabular}

Source : d'après Nicolau M., 2010

Pour autant, si la représentation d'une continuité totale est presque inexistante pour le corridor des étangs $(6 \%)$ - de fait entrecoupé de nombreuses routes -, elle concerne néanmoins un tiers des répondants du corridor du RER, voire plus de $40 \%$ de ceux de Champs, où la continuité s'avère pourtant moins « rectiligne » que dans l'Allée des Bois (RER).

\subsection{Mais les corridors font l'objet de perceptions sensorielles}

Mais si ni la dimension écologique de la biodiversité, ni la dimension spatiale de la continuité ne sont clairement perçues, faut-il pour autant conclure à une absence de lien entre l'attachement évoqué précédemment et la biodiversité existante? Plusieurs questions du questionnaire portaient sur les perceptions sensorielles des usagers dans ces espaces. Or ces perceptions, telles qu'elles ressortent de l'enquête, font apparaître une dimension écologique qui, pour n'être pas consciemment explicitée, n'en est pas moins 
bien présente dans l'intérêt porté à ces espaces. La présence d'animaux revêt ainsi un caractère important dans la mesure où pas moins de $88 \%$ de l'échantillon déclare voir des animaux. Si l'on retire les réponses relatives aux animaux domestiques tels que chiens et chats, ils sont encore $65 \%$ à mentionner des oiseaux, plus de $30 \%$ à citer différentes types de mammifères, $5 \%$ à mentionner des poissons et presque $3 \%$ à parler des insectes. Les usagers déclarent ainsi avoir vu en moyenne entre 2 et 3 animaux différents et citent au total près de 80 espèces. La diversité végétale est également perçue. Pour les arbres ce sont ainsi 70 \% des enquêtés qui disent en distinguer plusieurs espèces, la moitié d'entre eux étant capable d'en citer au moins deux. Les pourcentages sont légèrement inférieurs pour les herbes ( $52 \%$ des usagers ont repéré plusieurs espèces d'herbes) et ce sont au total près de 80 espèces herbacées qui sont nommées par l'ensemble des enquêtés.

Cette attention portée aux espèces animales et végétales participe, en outre, de l'attrait de ces espaces, et plus intéressant encore, de l'agrément que l'on peut y ressentir. $42 \%$ des enquêtés affirment entendre des sons agréables et $39 \%$ sentir des odeurs agréables. Or ce ressenti est directement lié à la biodiversité : les sons agréables proviennent pour l'essentiel des animaux (72\%), secondairement de la végétation ou de l'eau (13\%) alors que les odeurs dépendent avant tout du végétal (69\%). Sons et odeurs liés à la présence d'espèces animales et végétales participent donc du sentiment de satisfaction éprouvé dans ces corridors tout comme la qualité esthétique des lieux, citée parmi les mots qui décrivent le mieux ces espaces juste après le calme et la verdure déjà mentionnés.

On observe ainsi un profond décalage entre la connaissance de la biodiversité (pratiquement inexistante) et sa perception qui participe très largement de l'attrait ressenti pour ces espaces, sans que l'on puisse ici affirmer qu'une forte biodiversité entrainerait une augmentation des perceptions positives. Le rôle de ces perceptions sensorielles comme "porte d'entrée" vers une meilleure prise en compte de la biodiversité souligne au final l'importance des lieux considérés aussi bien sous l'angle de leur diversité biologique que sous l'angle de leurs qualités esthétiques et paysagères.

\subsection{La qualité écologique du lieu comme fondement de l'attachement à l'espace}

En dernier lieu, il nous semble que la qualité écologique du lieu est également un facteur important de l'attrait qu'exerce le lieu et de l'attachement qu'on y porte. Cette idée peut s'illustrer par le fait qu'un espace pauvre en biodiversité, tel « la liaison verte RER » (fig. 2), se traduit par un attachement faible au lieu. En l'occurrence, c'est là que les usagers interrogés se sentent le plus en ville, pratiquent le moins d'activités liés à la nature (comme "observer la nature»), et sont le moins favorables à une augmentation des impôts locaux, au paiement d'un ticket ou à un don en faveur de la protection et de l'entretien du corridor. L'attachement au corridor - où ils sont malgré tout présents pourrait être qualifié dans ce cas d'attachement à un espace public de proximité, et non d'attachement à ses aspects «naturels ».De fait, c'est bien dans ce corridor que les usagers qui habitent à moins de $10 \mathrm{mn}$ à pied et qui restent moins d'une heure sont les plus nombreux. À l'inverse, c'est dans les groupes fréquentant les corridors de Champs et des étangs qu'on observe le plus de pratiquants d'activités exclusivement liées à la nature (observer la nature et pêcher) et d'activités intermédiaires, et le plus de personnes qui se sentent en ces lieux soit «à la campagne », soit «à la fois en ville et à la campagne».Les 
usagers de ces deux corridors sont aussi proportionnellement plus nombreux à accepter un supplément d'impôts locaux ou le paiement d'un ticket ou d'un don.

Au-delà d'une comparaison simple entre corridors écologiques et «liaison verte » du RER, il est intéressant de mettre également en exergue des différences entre les deux corridors écologiques. Ainsi, c'est dans le corridor des étangs, le plus riche en biodiversité avérée (fig. 2), que l'attachement au corridor paraît le plus fort, comparativement au corridor de Champs-sur-Marne, dont la richesse en biodiversité n'est que "potentielle » (fig. 2). En effet, les activités non liées à nature sont proportionnellement les plus réduites dans le corridor des étangs, la fréquentation y est la plus longue, les personnes interrogées s'y sentent le plus « à la campagne ». C'est dans ce corridor également que la perception sensorielle des animaux, des arbres et des fleurs et herbes est la plus élevée et que les citations d'espèces différentes d'oiseaux sont les plus nombreuses. Richesse écologique et attachement ou intérêt pour le lieu sont là encore liés - quand bien même d'autres facteurs socio-économiques peuvent jouer dans cet attachement. On constate en effet que c'est dans le corridor de Champs-sur-Marne et non dans celui des étangs que le consentement à payer $\left(\mathrm{CAP}^{15}\right)$ est le plus important (qu'il s'agisse d'un don, d'un ticket d'entrée ou d'un supplément d'impôts). Il s'avère que c'est aussi dans ce corridor que les usagers disposent des revenus les plus élevés et résident le plus dans une maison, de surcroît avec jardin. Ces facteurs économiques et contextuels influent de toute évidence, eux aussi, sur le montant du consentement à payer - quand bien même nos analyses n'ont pas permis de le montrer ${ }^{16}$. De la même façon, on pourrait considérer que la possibilité ou l'impossibilité physique de se rendre dans un autre espace vert ou milieu naturel pourrait influer sur le consentement à payer - une forme de substitution que nous n'avons pas testée.

En troisième lieu, on peut souligner l'importance de la qualité écologique du lieu par une analyse du consentement à payer pour ce lieu, notamment par les populations les moins aisées qui sont a priori les moins enclines à financer la protection et la conservation de la biodiversité. On avait déjà observé qu'elles étaient paradoxalement les plus nombreuses à être favorables au paiement d'un ticket d'entrée, on constate en l'occurrence qu'elles le sont davantage encore dans un corridor de qualité écologique - celui de Champs-surMarne - (fig. 3) que dans un corridor dépourvu de ces qualités, celui du RER (fig. 4). Le consentement à payer des usagers est donc lui aussi en partie lié à la qualité écologique des lieux. 
Figure 3 : le consentement à payer (CAP) pour la protection du corridor de Champs-sur-Marne, en fonction des revenus (test du khi 2 significatif au seuil de $5 \%$ )

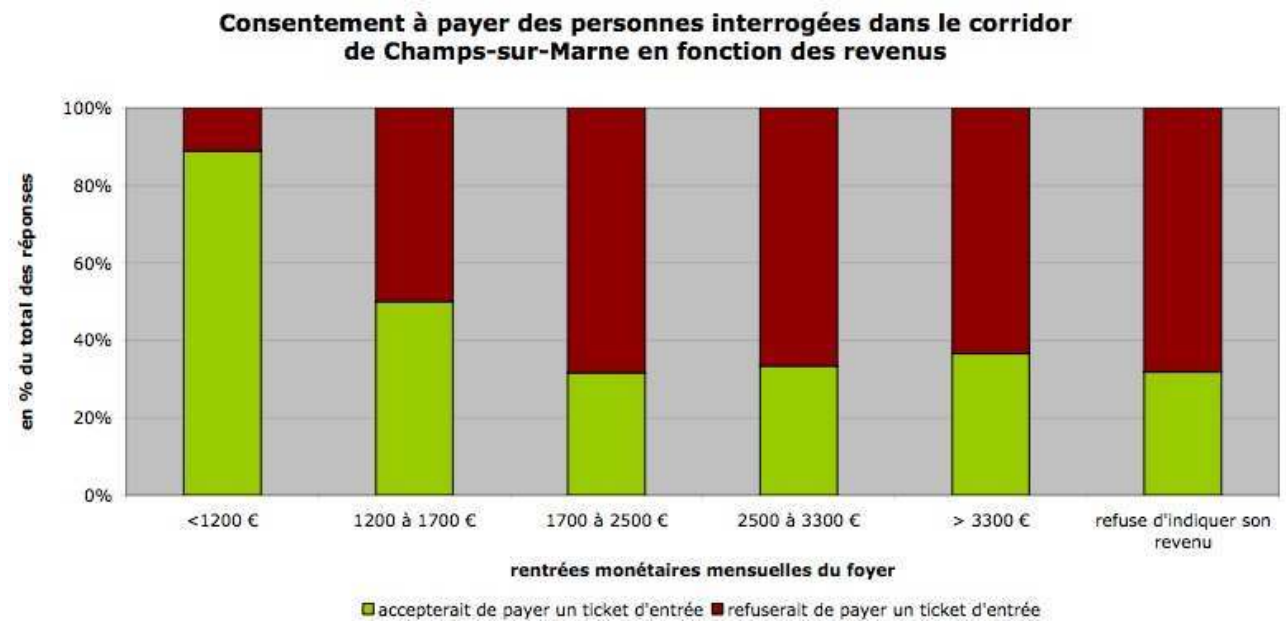

Figure 4 : le consentement à payer pour la protection du corridor du RER, en fonction des revenus (test du khi 2 significatif au seuil de $5 \%$ )

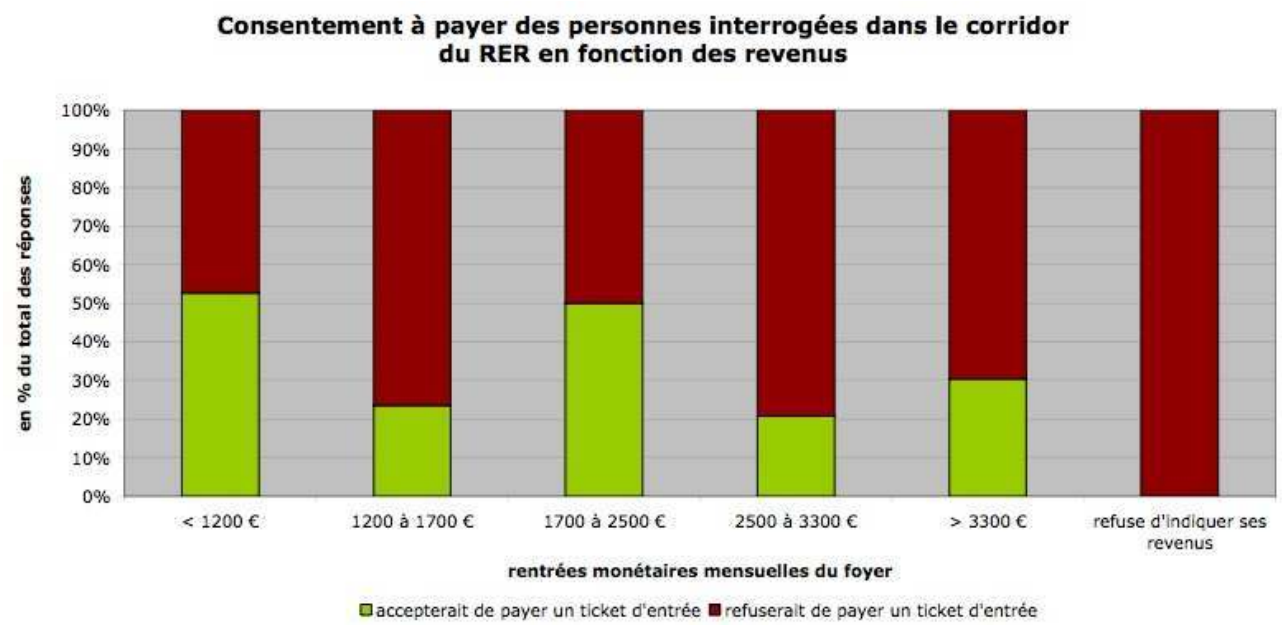

Sans qu'un lien direct puisse être établi entre biodiversité et bien-être des habitants, il apparaît cependant qu'une forte ${ }^{17}$ biodiversité existante contribue à la satisfaction des habitants en favorisant les perceptions positives et qu'un lien existe, sinon de causalité du moins indirect, entre attachement au lieu - que reflètent le CAP et la fréquentation - et qualité écologique, entendue dans sa double dimension écologique et paysagère.

\section{Discussion}

\subsection{Trame verte, biodiversité et « bien-être » des habitants}

Si l'établissement d'une relation entre biodiversité et bien-être des habitants ne saurait être établie par l'enquête que nous avons menée, plusieurs éléments nous semblent attester de l'intérêt porté par les habitants à la qualité écologique des lieux qu'ils fréquentent. La durée de fréquentation, que l'on peut considérer comme un indice d'attachement à l'espace, est ainsi bien supérieure dans le corridor des étangs possédant 
la qualité écologique la plus importante par rapport au corridor de Champs-sur-Marne et plus encore à la liaison verte du RER. Les sentiments agréables éprouvés constituent également des indices forts d'un lien entre qualité écologique du lieu et sentiment de satisfaction. Si les attentes des citadins concernent avant tout les services culturels, et donc l'espace vert plus que la diversité écologique s.s., c'est bien par l'intermédiaire de perceptions sensorielles directement liées à la diversité du vivant (animale et/ou végétale) - perceptions d'autant plus importantes que le nombre d'espèces identifiées augmente - que les habitants éprouvent un sentiment de bien-être. Le lien avéré entre diversité végétale et animale existante et perceptions de sons et d'odeurs agréables nous semble attester d'un lien indirect (car pratiquement jamais formulé) entre qualité écologique et sentiment de satisfaction. D'autres paramètres que la seule biodiversité spécifique (qualité esthétique et paysagère du lieu par exemple) peuvent entrer en ligne de compte dans cette relation à l'espace considéré. Il n'est donc pas possible en l'état d'affirmer un lien direct entre satisfaction des habitants et biodiversité.

\subsection{Un protocole d'enquête spécifique pour mesurer les liens entre biodiversité et bien être}

40 Il conviendrait pour cela de prolonger l'étude en essayant de déterminer si une augmentation de la biodiversité dans un espace donné peut se traduire par un accroissement des perceptions agréables et des sentiments positifs témoignant ainsi plus fortement d'un lien entre biodiversité et sentiment de bien-être. Une telle analyse suppose toutefois la mise en place d'un protocole d'enquête spécifique qui ne pouvait entrer dans cette étude et nécessiterait sans doute un partenariat avec des psychologues de l'environnement. Ce protocole consisterait en un long entretien réalisé sur le terrain, éventuellement avec l'aide d'un écologue. Il s'agirait de déterminer en premier lieu le degré de richesse de la biodiversité en divers lieux et de porter clairement cette réalité à la connaissance de la personne enquêtée, avant de lui demander de mesurer dans les lieux considérés son sentiment de bien-être sous forme de perceptions sensorielles de nature variée (olfactive, visuelle, auditive). Elle ferait donc directement par elle-même, et non plus indirectement, ce lien entre biodiversité et bien-être. Une enquête pourrait être conduite en parallèle dans les mêmes lieux en cherchant à faire mesurer par les enquêtés leur sentiment de bien-être sans leur indiquer le degré respectif de richesse de la biodiversité considérée. Ceci permettrait le cas échéant de tester l'importance de la connaissance de la biodiversité dans le sentiment de bien-être.

\subsection{Quelles implications en termes de politique paysagère ?}

Entreprendre une politique de valorisation des trames vertes auprès des habitants suppose toutefois d'associer une dimension paysagère à la prise en compte de la biodiversité. Le ressenti des habitants vis-à-vis de la biodiversité n'est en effet pas synonyme de perception des continuités écologiques et témoigne de la nécessité de réfléchir à une lisibilité paysagère de ces continuités et notamment des espaces de rupture. Les photographies 4 et 5 constituent des illustrations de ces ruptures, fréquentes dans les corridors du Val Maubuée, qui sont entrecoupés de routes ou de barrières visuelles, minérales comme végétales. Dans la photo 4 , l'Allée des Bois (corridor 2 ) venant ici du nord et se poursuivant vers le sud dans le bois de la Grange, a disparu du champ de 
vision, et n'est brutalement plus lisible en tant que corridor. Le bois présent à quelques dizaines de mètres - visible à l'arrière-plan de la photo - est à peine visible désormais, derrière les véhicules d'un parking récemment aménagé. Dans le corridor des étangs, ici à la hauteur de l'étang de l'écluse (photo 5), la continuité est également brutalement interrompue par une route en position dominante (le cours de l'Arche-Guédon). La circulation pédestre est certes maintenue, sous la route, mais le regard se heurte à une paroi verticale. Il n'est donc pas étonnant que les usagers n'aient pas de sentiment d'une cohérence écologique entre les étangs et les espaces végétalisés du corridor 3. Sans réflexion paysagère sur les moyens d'annuler ou d'atténuer ces coupures, ou de maintenir malgré elles une impression de continuité, une politique pédagogique de valorisation de la biodiversité perdrait de sa force. Continuité écologique et cohérence paysagère sont complètement liées dans les espaces linéaires de la trame verte urbaine.

Photographie 4 : la « liaison verte RER » (corridor 2).

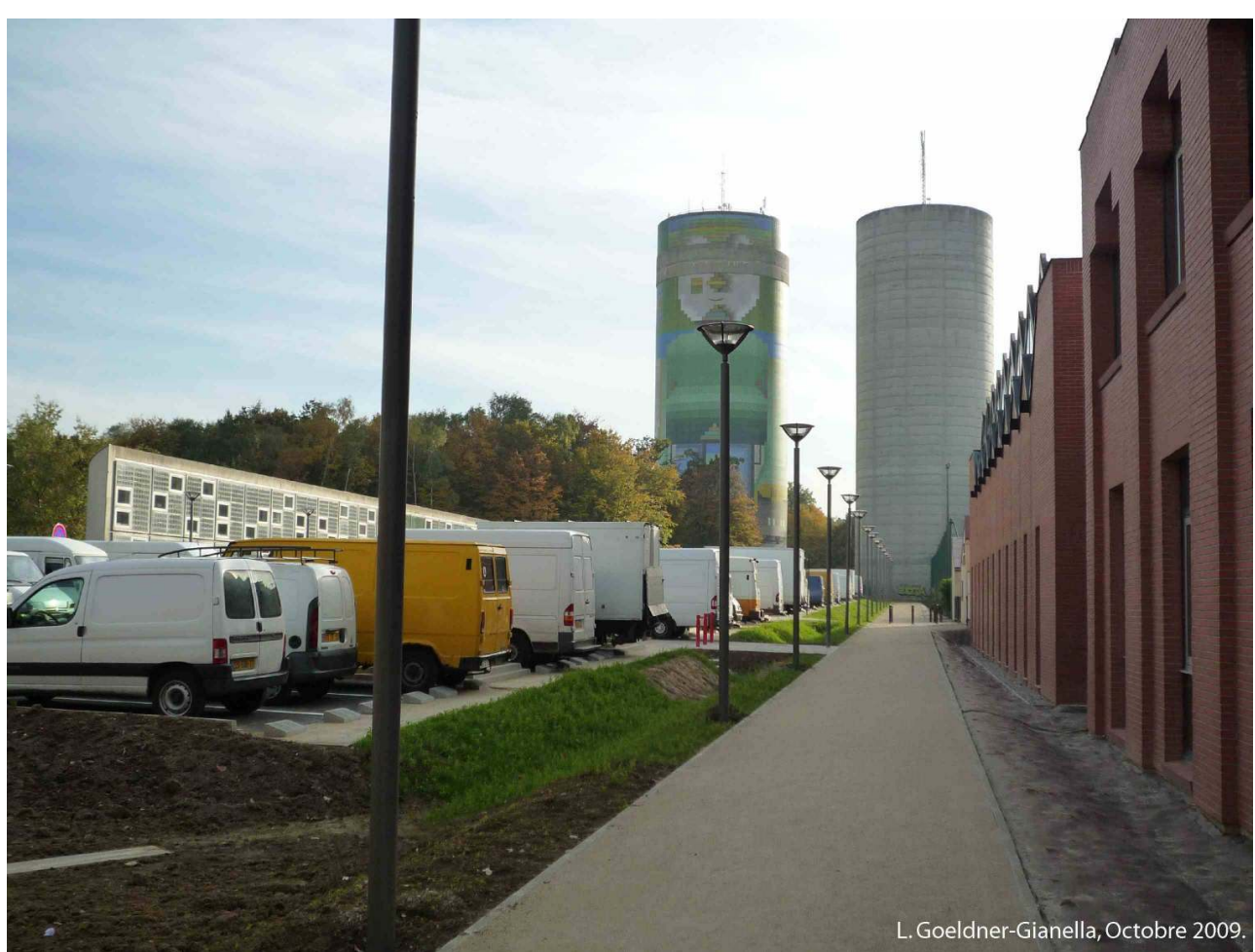


Photographie 5 : Une coupure dans le corridor des étangs.

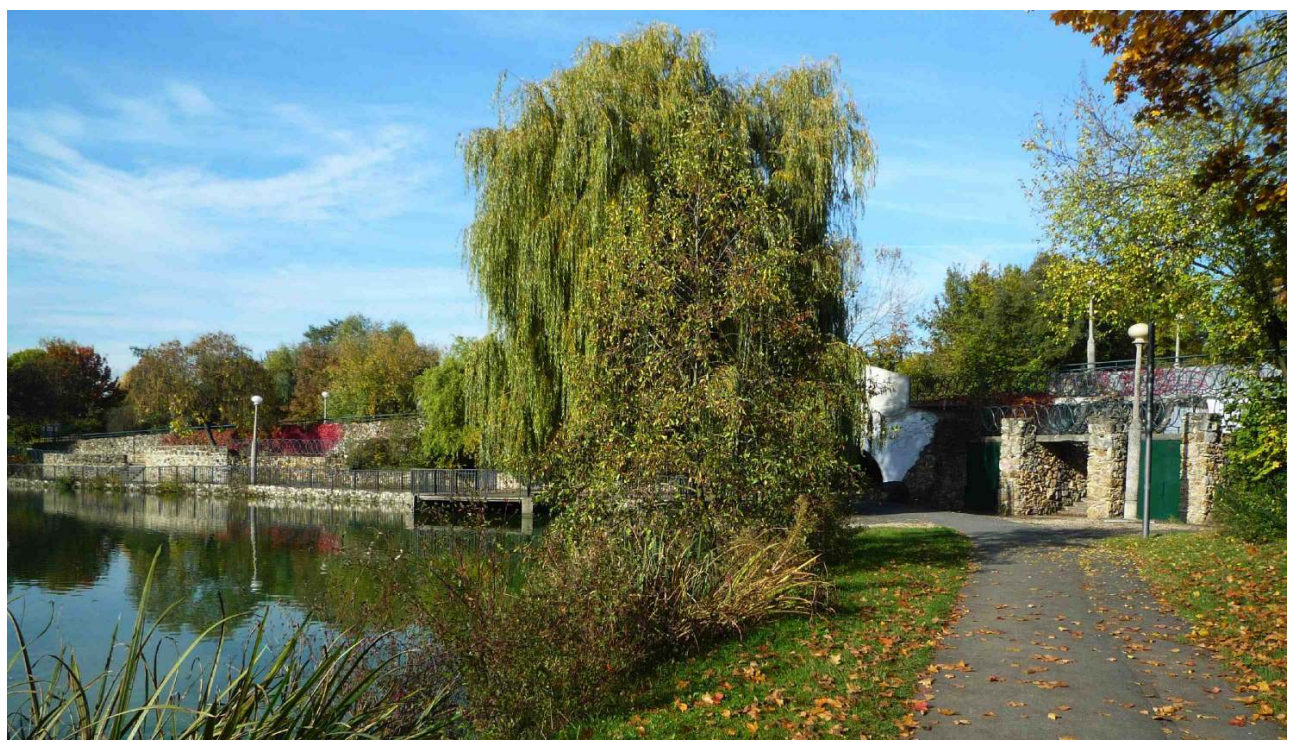

\section{Conclusion}

L'enquête menée sur la trame verte urbaine du Val Maubuée permet d'ouvrir quelques pistes de réflexion relatives aux politiques menées actuellement en faveur de la biodiversité urbaine, et plus particulièrement aux politiques de mise en place des trames vertes urbaines. Ces politiques de mise en place des trames vertes ne sauraient faire l'impasse sur la dimension sociale de cette relation aux espaces de biodiversité intraurbains. L'un des principaux enseignements de l'étude réside dans l'attachement de citoyens, par ailleurs souvent peu reliés aux espaces de biodiversité et peu conscients des enjeux, à ces espaces intra-urbains. La fréquentation des corridors étudiés ne reproduit pas les inégalités sociales mises en évidence dans une précédente enquête et qui concernaient la connaissance de la biodiversité et surtout l'accès aux espaces de biodiversité. La coupure sociale et générationnelle que nous avions mise en évidence ne se retrouve pas ici. Ces espaces reflètent même un attachement relativement plus fort de la part de populations aux revenus modestes : le consentement à payer pour la protection et la préservation de ces espaces est en effet proportionnellement plus fort dans des catégories sociales peu favorisées. Les politiques en faveur de la biodiversité urbaine ne sauraient donc se baser uniquement sur l'amélioration de la qualité écologique et paysagère des lieux, mais aussi sur l'amélioration de l'offre en espaces de biodiversité en faveur de ces populations. 


\section{BIBLIOGRAPHIE}

Arrif T., Blanc NClergeau P., 2011, « Trame verte urbaine, un rapport Nature-Urbain entre géographie et écologie », Cybergéo, document 574, http://cybergeo.revues.org/24862.

Berger M., 2004, Les périurbains de Paris. De la ville dense à la métropole éclatée ?, Paris, CNRS Éditions, Espaces et Milieux, $317 \mathrm{p}$.

Blanc, N., Clergeau, P. 2010, « Installer une trame verte dans la ville? Le point de vue des chercheurs? ", Numéro spécial d'Urbanisme "La démarche Écocité. Villes durables en projet", Hors série $\mathrm{n}^{\circ} 36$, pp. 55-59

Cincotta R. P., Wisnewski J., Engelman R., 2000, « Human population in the biodiversity hotspots ", Nature, 404, p. 990-992.

Dearborn, D. C., and Kark S.,. 2010, Motivations for Conserving Urban Biodiversity. Conservation Biology 24 :432-440.

Devictor, V., Julliard R., JiguetF. 2008, "Distribution of specialist and generalist species along spatial gradients of habitat disturbance and fragmentation",- Oikos, $117:$ p. 507-514

Filoche S., Perriat F., Moret J., Hendoux F2010, Atlas de la flore sauvage de Seine-et-Marne? Deauville, Editeur Librairie des musées. Conseil General de Seine-et-Marne, 687 p.

Eleb-Harlé N., Barles S. (dir.), 2005, Hydrologie et paysages urbains en villes nouvelles, rapport final, Octobre 2005, METLM, Programme d'histoire et d'évaluation des villes nouvelles françaises, 118 p., http://www.cdu.urbanisme.equipement.gouv.fr/IMG/pdf/rapportfinal.pdf

Fuller, R. A., Irvine K. N., Devine-Wright P., Warren P. H., Gaston K. J., 2007, « Psychological benefits of greenspace increase with biodiversity », Biol. Lett., 3, p. 390-394. Published online 15 May 2007.

Lardinois R., 2010, Étude de la fréquentation de corridors écologiques, Rapport de projet tutoré, L3 professionnelle Géomatique et Environnement, Université Paris 1, 15 p.

Menozzi M.-J., 2011, Les espaces verts du Val Maubuée. Analyse de la fréquentation, rapport d'expertise pour le San du Val Maubuée, 50 p.

Mimet A., Simon L., Julliard R., 2011, "Giving back fragmentation its importance on biodiversity dynamics", European Ecological Federation, Avila, Spain, septembre 2011.

Nicolau M., 2010, Les perceptions, les pratiques et les représentations sociales dans les corridors du Val Maubuée, Mémoire de Master 1, Université de Paris 1, 138 p.

Shwartz A., Cosquer A., JaillonA., PironA, JulliardR., RaymondR., SimonL., Prévot-Julliard A.C. (2012) Urban Biodiversity, City-Dwellers and Conservation: How Does an Outdoor Activity Day Affect the Human-Nature Relationship ? PLoS ONE vol. 7, issue $6:$ http://dx.plos.org/10.1371/ journal.pone.0038642

Simon L., Riboulot M., Goeldner-Gianella, L., Humain Lamour A.L., 2012, « La biodiversité perçue et vécue par les urbains en Seine-et-Marne », in Prévost-Julliard A.C., Fleury C. (dir.), L'exigence de la réconciliation. Biodiversité et société, Paris, Fayard.

Théobald D. M., 2001, "Land-use dynamics beyond the American urban fringe", in Geographical Review, 91, p. 544-564. 
Teyssèdre A., 2004, « Vers une sixième grande crise d'extinctions ? ", in Barbault R., Chevassusau-Louis B. (dir.), Biodiversité et changements globaux. Enjeux de société et défis pour la recherché, Edition adpf, p. 24-36.

Vitousek P.M., Mooney H.A., Lubchenko J., Melillo J.M., 1997, « Human domination of earth's ecosystems ", Science, 277, p. 494-499.

Wilcove D. S., Rothstein D., Dubow J., Phillips A., Losos E., 1998, « Quantifying threats to imperiled species in the United States » Bio-Science 48, p. 607-615.

Wittemyer G., Elsen P., Bean W. T., Burton A. C. OBrashares., J. S., 2008, « Accelerated human population growth at protected area edges ». Science, 321, p. 123-126.

Remerciements : Le travail présenté dans cet article est le résultat de recherches menées et financées dans le cadre du programme ANR Trame Verte Urbaine.

\section{NOTES}

1. SDRIF : Schéma Directeur de la Région Île-de-France; SCOT: Schéma de Cohérence Territoriale.

2. La notion de well-being englobe bien au-delà du simple bien-être des aspects beaucoup plus étendus touchant à la santé physique et psychologique, aux capacités d'interaction entre individus, etc...

3. Le projet EUROSCAPES est cofinancé par l'Union Européenne par le Fond Européen de Développement Régional à travers le Programme INTERREG IVC. Il a pour objectif d'améliorer la gestion des espaces et paysages naturels et culturels dans les zones urbaines et périurbaine.

4. Le terme de corridor est ici employé par commodité pour désigner les éléments de la trame verte urbaine étudiés.

5. www.meilleursagents.com/prix-immobilier/seine-et-marne-77/ Consulté le 27 Mars 2012.

6. Îlot Regroupé pour l'Information Statistique.

7. Des comptages sur photographies aériennes rapprochées ne sont pas plus envisageables du fait de la forte couverture végétale des corridors.

8. Plus de $70 \%$ des usagers des trois corridors résident dans les trois communes concernées de Champs-sur-Marne, Noisiel et Torcy.

9. Sur la base de la population âgée de 15 ans et plus, la seule prise en compte dans notre enquête.

10. La mesure globale de la fréquentation de ces espaces se heurte à la multiplicité des lieux de passage et plus encore à la variabilité temporelle (hebdomadaire et saisonnière notamment) des usages de ces espaces.

11. D'un montant allant de 1 à 750 euros, selon les usagers.

12. D'un montant allant de 50 centimes à 10 euros, selon les usagers.

13. D'un montant de 10 à 300 euros, selon les usagers.

14. C'est-à-dire entre le parc du château de Champs-sur-Marne et le bois de la Grange (corridor de Champs), entre le parc de Noisiel et ce même bois (corridor du RER) ou entre la Marne et le RER de Lognes (ou le parc du grand bassin), pour la branche sud-ouest du corridor des étangs, ou l'étang de Segrais-sud pour sa branche sud-est.

15. CAP (consentement à payer) : méthode utilisée en sciences économiques pour déterminer le prix d'un bien ou d'un service hors marché.

16. Nous avons croisé le consentement à payer (ticket, don, impôts) avec le type de situation résidentielle de chaque enquêté (maison avec jardin, sans jardin, immeuble avec ou sans jardin), sans faire ressortir de relation significative entre ces deux variables. 


\section{RÉSUMÉS}

Une première enquête, menée sur 4 communes périurbaines de Seine-et-Marne concernant les relations des habitants à la biodiversité, soulignait l'importance des espaces de proximité dans le lien qui unit les habitants à la biodiversité. Cette même enquête mettait en évidence les différenciations sociospatiales (Catégories Socio-Professionnelles, niveau de diplôme, type d'habitat...) qui existent dans le rapport à la biodiversité. Le présent article s'interroge, à la suite de ce premier travail (Simon et al, 2012), sur le rôle que peuvent jouer les trames vertes urbaines dans la connexion des habitants à la diversité du vivant : espaces intra-urbains, les trames vertes sont-elles les lieux d'une relation particulière des habitants à la biodiversité et si oui à quelle biodiversité? Espaces de fréquentation locale dominante, la trame verte permet-elle de connecter à la biodiversité des populations autrement sans lien avec la « nature »?

Biodiversity, inhabitants and urban green infrastructures : the Val Maubuée case study (Seine-etMarne, France). A first survey leaded in fourth suburban townships of the Department of Seineet-Marne (France) concerning the associations between inhabitants and biodiversity underlined the importance of spatial proximity for connecting people to biodiversity. The same survey put emphasis on the social and spatial differences concerning this relationship. Consequently, this article questions the role that urban green infrastructures can play by connecting people to biodiversity: are urban greenways places of a particular relationship between inhabitants and biodiversity? If so, to what kind of biodiversity? As a space more frequently used by local people, do the urban green infrastructure be a innovative way of connecting to biodiversity the groups who have very few links with "nature"?

\section{AUTEURS}

\section{LAURENT SIMON}

Laurent Simon est Professeur de géographie à l'Université Paris 1 - Panthéon Sorbonne, HESAM. Spécialiste de biogéographie, il est membre du laboratoire LADYSS. Il a déjà écrit ou contribué à plusieurs ouvrages, dont la Géographie de l'environnement, Belin, 2007 et L'Atlas des forêts dans le monde, Autrement, 2009. Ses travaux actuels portent notamment sur les questions de biodiversité en ville et dans les espaces forestiers européens.

\section{LYDIE GOELDNER-GIANELLA}

Lydie Goeldner-Gianella est Maître de conférences en géographie à l'Université Paris 1, habilitée à diriger des recherches, membre de l'UMR 8586 PRODIG. Ses travaux portent sur la perception et les représentations sociales dans le domaine de l'environnement (risques naturels, espaces naturels, biodiversité), notamment approchées au moyen des techniques d'enquête dont elle a souligné les spécificités et difficultés en ce domaine (L'Espace géographique, 2010/4). 